\title{
Runaway electron energy measurement using hard x-ray spectroscopy in "Damavand" tokamak
}

\author{
C. Rasouli, ${ }^{1}$ D. Iraji, ${ }^{2}$ A. H. Farahbod, ${ }^{1}$ K. Akhtari, ${ }^{3}$ H. Rasouli, ${ }^{1}{ }^{H}$. Modarresi, ${ }^{4}$ and \\ M. Lamehi ${ }^{1}$ \\ ${ }^{1}$ School of Plasma Physics and Nuclear Fusion, Institute of Nuclear Science and Technology, AEOI, \\ P.O. Box 14155-1339, Tehran, Iran \\ ${ }^{2}$ Centre de Recherches en Physique des Plasmas, Association EURATOM-Confédération Suisse, \\ Ecole Polytechnique Fédérale de Lausanne, CH-1015, Switzerland \\ ${ }^{3}$ Department of Physics, Sharif University of Technology, P.O. Box 11365-9161, Tehran, Iran \\ ${ }^{4}$ Department of Mechanical Engineering, Sharif University of Technology, P.O. Box 11365-9567, Tehran, Iran
}

(Received 10 July 2008; accepted 14 December 2008; published online 16 January 2009)

\begin{abstract}
Set of experiments has been developed to study existing runaway electrons in "Damavand" tokamak plasma upon characteristics of hard x-ray emissions produced by collision of the runaway electrons with the plasma particles and limiters. As a first step, spatial distribution of hard $\mathrm{x}$-ray emissions on the equatorial plane of the torus was considered. Obtained spectra of hard x-ray emissions for different alignments of shielded detector indicate isotropic emissivity in the equatorial plane. This is in agreement with wide angle cone of bremsstrahlung radiations, deduced from the mean value of energy of the runaway electrons. The mean energy was calculated from the slope of the energy spectrum of hard x-ray photons. In the second stage in order to investigate time evolution of energy of the runaway electrons, similar technique were applied to obtain hard x-ray energy in every $3 \mathrm{~ms}$ intervals, from the beginning to the end of plasma. The mean energy of the runaway electrons increases during the ramp up phase and reaches its maximum between 3 and 9 ms after plasma formation. Also considering the time dependence of the counted photons in each energy range shows that energetic photons are emitted during the ramp up phase of the plasma current in Damavand tokamak. (C) 2009 American Institute of Physics. [DOI: 10.1063/1.3064917]
\end{abstract}

\section{INTRODUCTION}

Electrons in tokamak plasma with energies higher than critical level are continuously accelerated by the toroidal electric field, i.e., they run away. Runaway electrons represent a major threat in tokamak machines due to the damage produced when they hit the first wall. ${ }^{1}$ Also the generation of runaway electrons and their effect on behavior of ionized and neutral particles, in particular, and plasma characteristics in general have been the matter of speculation by many researchers. In order to study such a phenomenon, a variety of models have been proposed that are intended to predict different behaviors of the runaway electrons in the future tokamaks. However, so far two dominant mechanisms have been proposed to describe generation mechanism of the runaway electrons in tokamak plasma. One is the "conventional runaway mechanism" and the other is the "avalanche runaway mechanism."

Conventional runaway mechanism was first discovered by Dreicer ${ }^{2}$ in 1959 so it is also known as the "Dreicer mechanism." According to this model if the applied electric field exceeds $E_{D}$, where

$$
E_{D}=n_{e} e^{3} \ln \Lambda / 4 \pi \varepsilon_{0}^{2} T_{e},
$$

which is called the Dreicer electrical field, ${ }^{2}$ thermal electrons will start to run away ${ }^{3}$ and they may go out of the plasma and cause energy loss. Conventional or Dreicer runaway electrons finally reach a relativistic saturation speed predicted upon relativistic dynamics equations. ${ }^{4}$ Another process which is called avalanche mechanism was proposed by Sokolov. ${ }^{5}$ It happens when applied electric field exceeds $E_{c}$, where

$$
E_{c}=T_{e} E_{D} / m_{e} c^{2}
$$

is the critical electric field required to initiate avalanche mechanism. Despite the fact that in an avalanche runaway mechanism applied electric field is less than the Dreicer electric field, i.e., $E<E_{D}$, a few numbers of existing runaway electrons increase the energy of thermal electrons due to close collisions and bring them to runaway regime while themselves stay still in runaway regime. Repetition of this mechanism may cause gradual conversion of plasma current into runaway current. ${ }^{6}$

In large scale tokamaks at the disruption moments production of runaway electrons and their second influences on plasma is very important. ${ }^{7-9}$ Collision of intense runaway electrons with the first wall would significantly reduce the lifetime of the first wall in fusion reactors. Therefore control and annihilation of runaway currents for the future reactors have been considered by researchers and several methods such as plasma current ramping up, controlled inward plasma shifting, and safety factor decreasing, have been found in JT-60U. ${ }^{10}$ However, to obtain deeper knowledge about runaway electrons, more investigations considering different aspects of runaway electrons such as electron cyclotron emission (ECE) and bremsstrahlung radiations are carried out. 

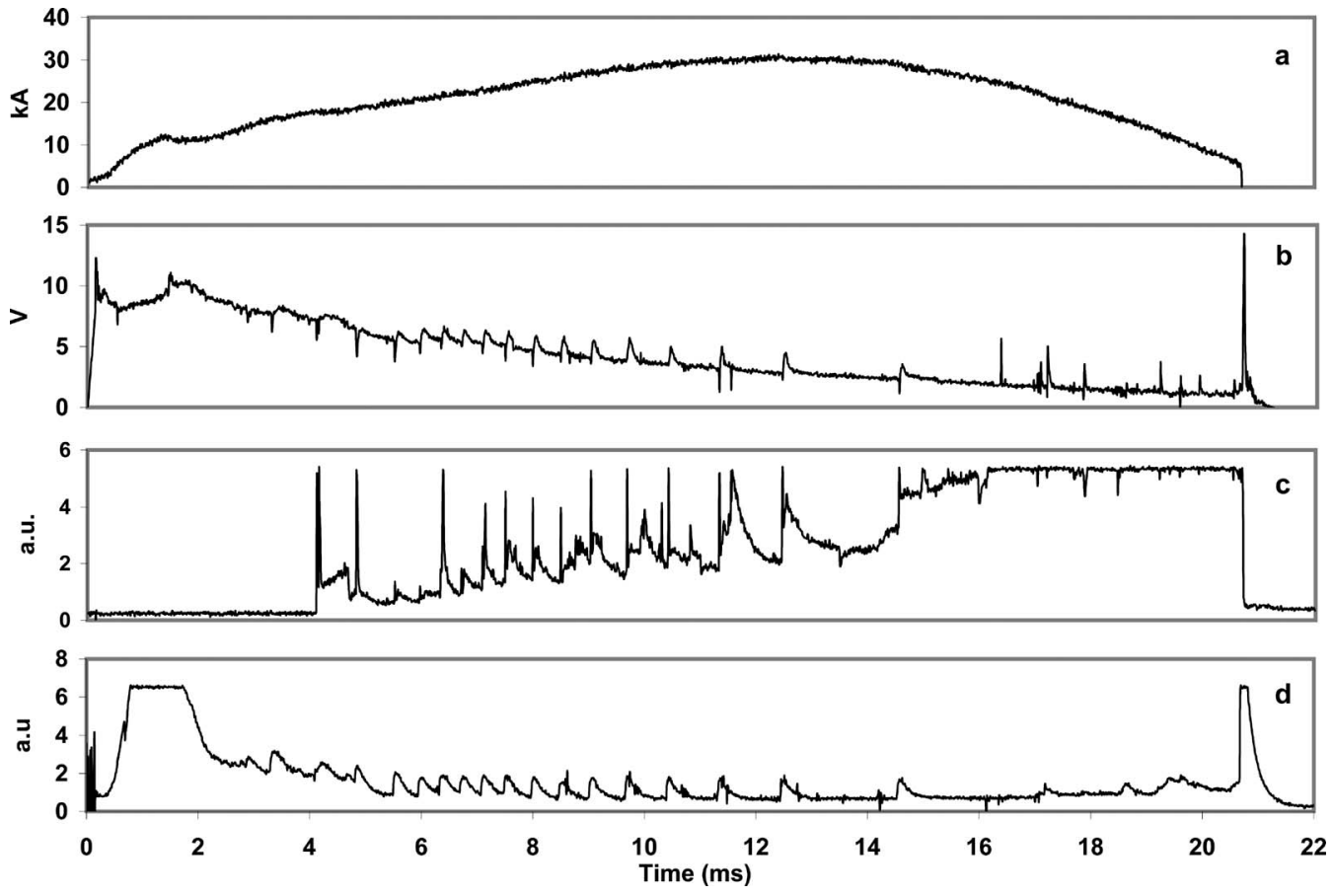

FIG. 1. (a) Plasma current, (b) loop voltage, (c) hard x-ray intensity, and (d) hydrogen line radiation intensity at $4860 \AA$ with respect to the time during a typical shot.

Due to collisions of energetic runaway electrons with plasma ions and tokamak limiter, bremsstrahlung radiations in the form of hard x-ray photons are emitted from the plasma and the limiter, which may provide valuable information about plasma and runaway electrons. ${ }^{11,12}$

So far in different tokamaks, many experiments have been done to deduce energy of runaway electrons from the energy spectrum of hard x-ray photons. ${ }^{13,14}$ Damavand tokamak is a small size machine, which could provide convenient conditions to investigate the mechanisms of production, behavior, prevention, and shutdown of runaway currents in fusion plasmas. As a first step to study hard x-ray emissions in Damavand plasma, behavior of radiated hard $\mathrm{x}$-ray after major disruption has been investigated. ${ }^{15}$

Collisions of energetic electrons with the residual plasma particles and the first wall are two sources for hard x-ray radiations due to the runaway electrons in tokamak plasma. Although the intensity of radiations due to collisions with heavy nuclei of the first wall is higher, however, in both cases dominant mechanism is bremsstrahlung. According to bremsstrahlung mechanism, most of hard x-ray photons are emitted in a cone with the half angle of $1 / Y$ in the direction of the velocity of the emitting runaway electrons. ${ }^{8} Y$ is obtained by

$$
Y=\frac{E_{r}(\mathrm{MeV})}{0.512} .
$$

Also the energy distribution function of runaway electrons can be described as

$$
f(E) \propto \exp \left(-\frac{E}{E_{r}}\right),
$$

where $E$ is the energy of hard x-ray photons and $E_{r}$ is the mean energy of runaway electrons. Therefore $E_{r}$ could be deduced from the inverse slope of the energy spectrum $^{16,17}$ and, consequently, $Y$ is calculated.

In this work, NaI-scintillator detectors have been used to measure and to analyze hard x-ray spectra of Damavand tokamak radiations in different directions and in different times during plasma establishment. So for each alignment of detectors, considering Eqs. (3) and (4), the mean energy of runaway electrons was calculated from the inverse slope of hard x-ray spectra and then $Y$ was obtained.

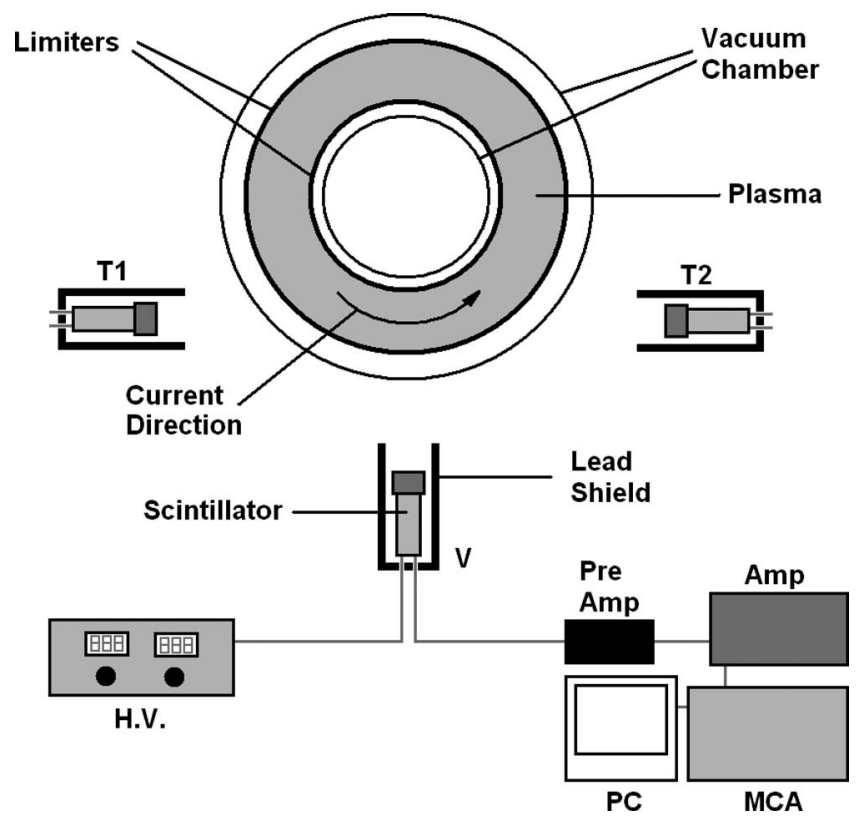

FIG. 2. Schematic of the experimental setup: V is the scintillator perpendicular to plasma current, $\mathrm{T} 1$ is viewing toward the direction of the plasma current and T2 in the opposite direction of the plasma current, Pre Amp and Amp are, respectively, the preamplifier and the amplifier, $\mathrm{HV}$ is high voltage power supply of scintillator, and MCA is Multichannel Analyzer. 

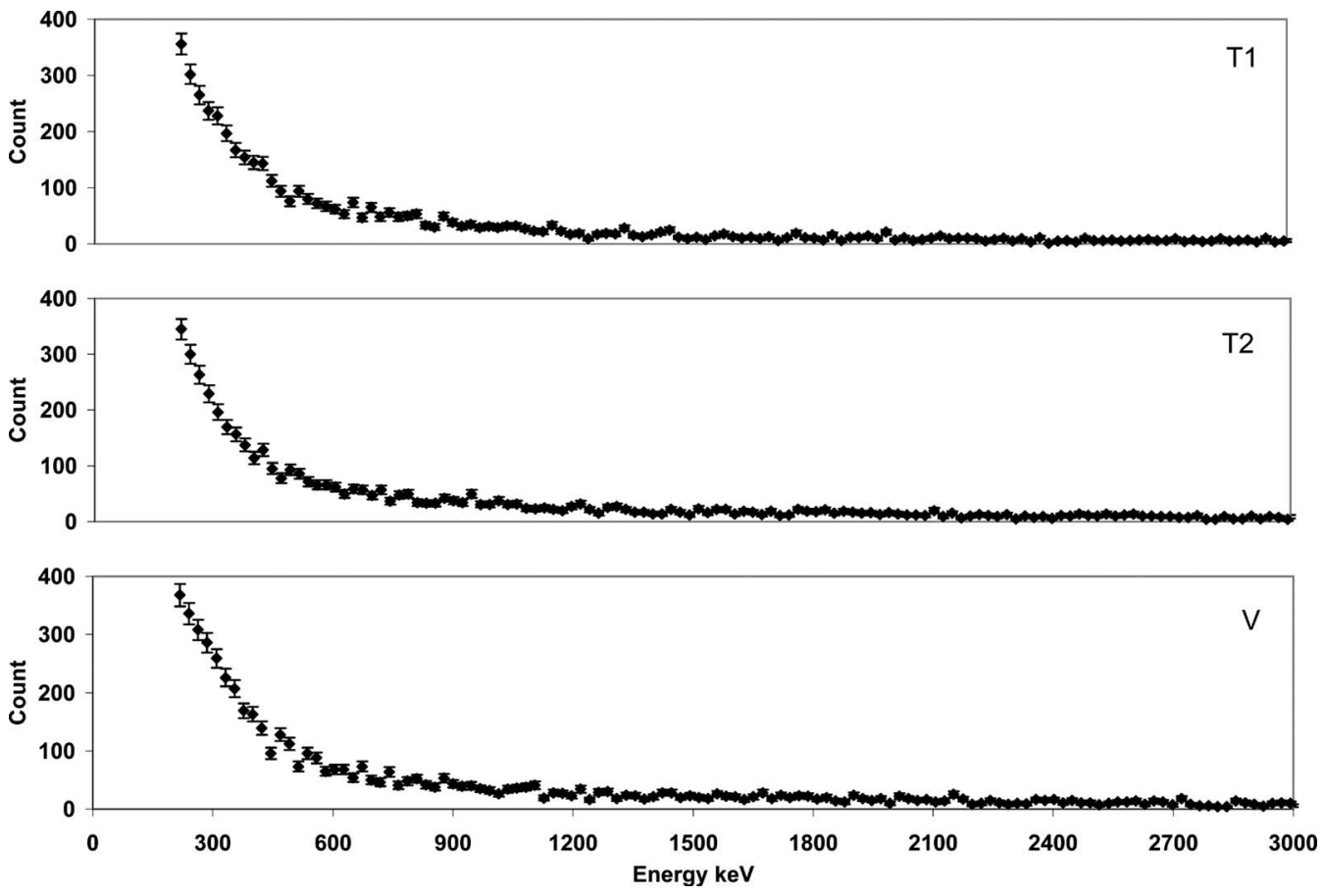

FIG. 3. Hard x-ray emission energy spectra with respect to detector position and alignment at the equatorial plane of tokamak. T1 is tangential, which is in the direction of plasma current, T2 is tangential, which is opposite to the direction of plasma current, and V is perpendicular to the plasma current.

\section{EXPERIMENTS}

All experiments addressed in this article have been done on Damavand tokamak, which is categorized as a small size tokamak. The main characteristics of Damavand tokamak are as follows: the main radius is $R=36 \mathrm{~cm}$ and it has an elongated cross section having the parameters $b=10 \mathrm{~cm}$ and $a$ $=7 \mathrm{~cm}$. This Tokamak has a toroidal magnetic field of $B_{T}$ $=1.2 \mathrm{~T}$ and normally operates with plasma currents of about $I_{P}=28$ to $32 \mathrm{kA}$. Plasma duration is about $21 \mathrm{~ms}$ and typical values of electron and ion's temperatures are, respectively, $T_{e}=300 \mathrm{eV}$ and $T_{i}=150 \mathrm{eV}$. Damavand tokamak is equipped with a magnetron rf source in order to preionize the neutral gas before the main discharge. Furthermore, this tokamak has an active feedback system of $R$ and $Z$ position control. Figure 1 shows typical behavior of the plasma current, loop voltage, hard $\mathrm{x}$-ray emissions, and $\mathrm{H}$-beta radiations with respect to the time during plasma formation.

To detect hard $\mathrm{x}$-ray photons, $3 \times 3$ in. ${ }^{2}$ Bicron NaIscintillator detector, as well as a Canberra 8180 multichannel analyzer (MCA), was used. To obtain enough counts per channel, MCA was set to the "512 channel" mode. Two standard $\mathrm{Co}^{60}(1.17$ and $1.33 \mathrm{MeV})$ and $\mathrm{Cs}^{137}(0.66 \mathrm{MeV})$ sources were employed together in order to calibrate MCA in the range of interest energies of this work. Using a digital delay generator, MCA was run during a selected time window of the plasma establishment period.

To study of the spatial distribution of hard x-ray emissions as shown in Fig. 2 three scintillators ("V," "T1," and "T2") were positioned at $50 \mathrm{~cm}$ far from the vacuum vessel at the equatorial plane of the tokamak. Detector V was perpendicular to the vessel wall and detectors T1 and T2 were aligned tangential to the direction of the plasma current while T1 was viewed toward the direction of the plasma current and T2 in the opposite direction of the plasma current. All detectors were encapsulated in a lead cylinder to limit the field of view to less than $10^{\circ}$ in such a way that only those photons that were emitting along the direction of the detectors could be counted. However, consequently during one shot, MCA was not able to count enough photons to produce energy spectrum. So to obtain reasonable spectra, counts of ten successive and identical shots were added together. Then to exclude the background counts of each channel, using the same method, spectrum of the background radiations was obtained and was subtracted.

Besides spatial distribution of hard x-ray emissions, evolution of the energy spectrum and ultimately variation in the energy of the runaway electrons were considered as the next experiment, which always have been among the most addressed issues in terms of research of tokamak plasmas. So, complementary experiments were set to acquire the energy spectrum of hard x-ray emissions during shorter periods. According to preliminary tests, the whole $21 \mathrm{~ms}$ of the plasma duration was divided to seven intervals of $3 \mathrm{~ms}$ and using the signal generator, MCA was used to produce the energy spectrum of hard $\mathrm{x}$-ray radiations during a selected time window among the seven time windows. Of course, as mentioned before, obtained counts of ten identical shots were added to have clear spectrum of each interval. Following this method made it possible to have seven different energy spectra corresponding to the seven time intervals covering the duration of the plasma establishment. In this case an unshielded NaIscintillator detector was placed at $14 \mathrm{~m}$ far from the vessel on the equatorial plane to detect hard x-ray photons emitted from the entire tokamak. 

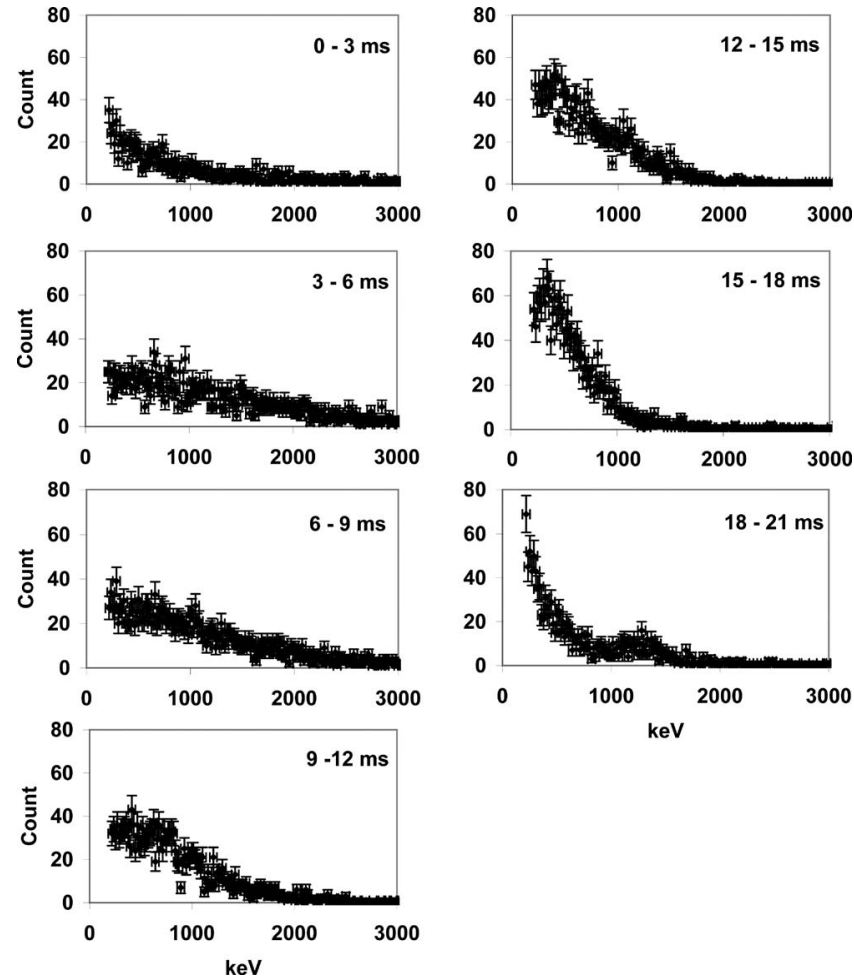

FIG. 4. Hard x-ray radiation spectra of seven time intervals of $3 \mathrm{~ms}$, covering entire plasma establishment duration.

\section{RESULTS}

Obtained hard X-ray spectra with respect to detector position are shown in Fig. 3. The error of counts for every channel is represented by a vertical bar. These errors indicate that all three spectra are almost similar and there is no considerable difference between their values and shapes.

Estimation of the mean energy of runaway electrons also
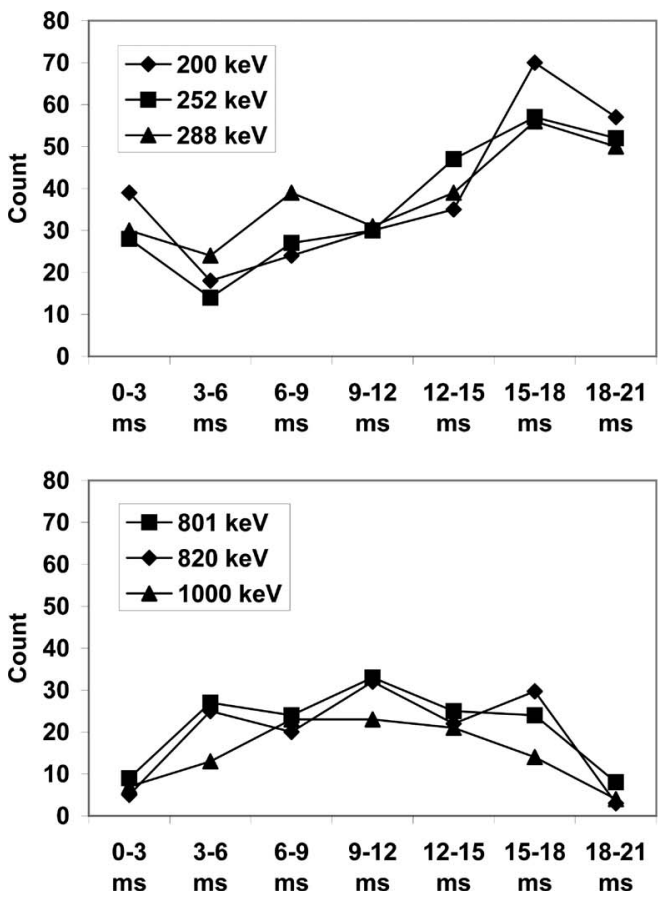

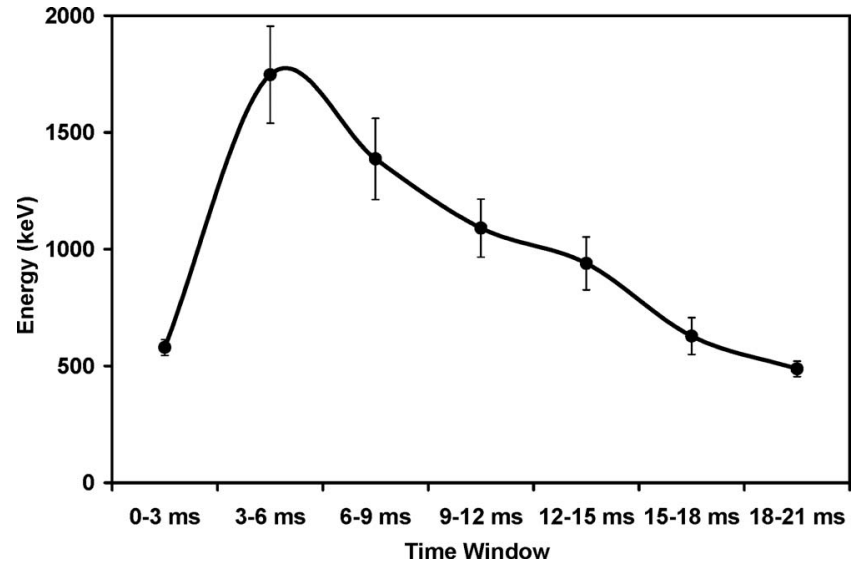

FIG. 5. Time evolution of the mean kinetic energy of runaway electrons during plasma establishment. From start of plasma the mean energy of runaway electron increases, there is an energy peak in 3-6 ms, and after that the mean energy gradually decreases.

leads to a similar result for the three different alignments of detectors $\mathrm{T} 1, \mathrm{~T} 2$, and $\mathrm{V}$, which represent respectively, directions tangential (indirection), tangential (antidirection), and perpendicular to the plasma current. Calculating $Y$ from $E_{r}$ results in a very wide angle of cone of bremsstrahlung radiation in Damavand tokamak. This confirms isotropic emissions of hard $\mathrm{x}$ rays at the equatorial plane of Damavand tokamak. Although the half-angle of the radiation cone for the electrons having energies more than $1 \mathrm{MeV}$ is small, very few of these runaway electrons have been detected. Hence the difference between the spectra, obtained from different alignments of detectors, is not considerable.

Figure 4 shows seven spectra of hard x-ray radiations corresponding to seven time windows from the start up of the plasma to the end. According to obtained spectra in Fig. 4 , the mean energy of runaway electrons was calculated for
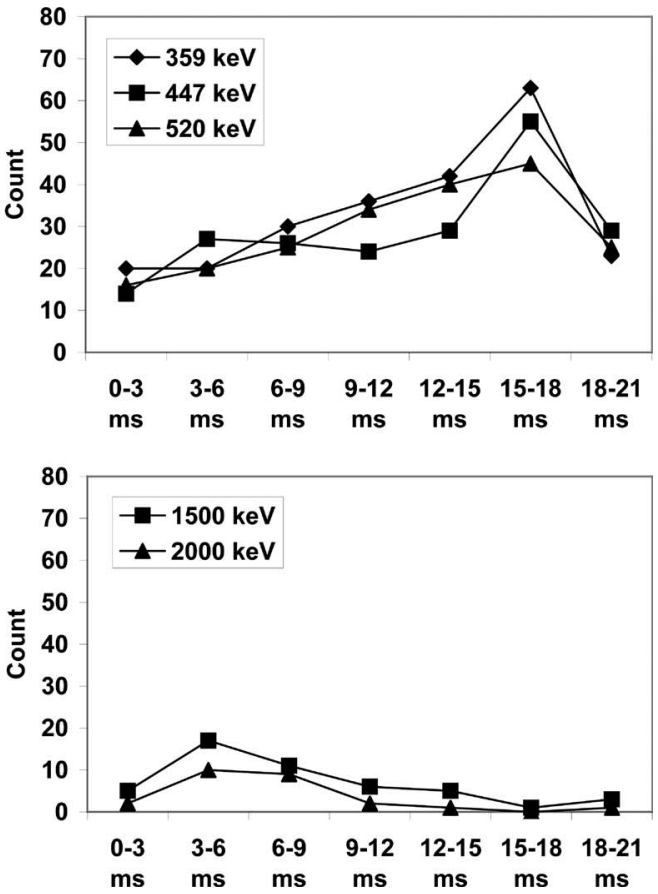

FIG. 6. The time evolution of the number of hard x-ray photons corresponding to several energies. 
each spectrum and the result is shown in Fig. 5. It is clear that there is a peak of the mean energy of runaway electrons during the 6-9 ms interval. This is in agreement with the fact that during the ramp up phase of the plasma current, the loop voltage is high enough and runaway electrons gain more energy from the electric field and reach their maximum values.

Also considering the time evolution of hard $\mathrm{x}$-ray radiations, having certain energy would be interesting. The time evolutions of the number of counted hard x-ray photons are shown in Fig. 6 for several energy ranges.

These curves show the time variation in the intensity of hard s-ray emissions, having different energies during shot. It indicates that during the first $3 \mathrm{~ms}$ the number of photons with energies from 200 to $300 \mathrm{keV}$ is larger and during 3-6 ms, a relative reduction of photons count is found. However, after this, a smooth increase in photon count is obtained until the end of plasma. This is while the counted photons containing energies ranging from 350 to $600 \mathrm{keV}$ have an incremental behavior. The number of counted photons ranging from $700 \mathrm{keV}$ to $1 \mathrm{MeV}$ has its peak almost during the same time as the peak of plasma current and later it shows a declining behavior. Photons having energies from 1.5 to $2 \mathrm{MeV}$ are detected only during 3-9 ms, which are coincident with the time that runaway electrons reach their maximum energy and later because of the energy reduction in runaway electrons to less than $1 \mathrm{MeV}$; consequently, high energy photons are not detected until the end of plasma.

${ }^{1}$ R. Yoshino, S. Tokuda, and Y. Kawano, Nucl. Fusion 39, 151 (1999).

${ }^{2}$ H. Dreicer, Phys. Rev. 115, 238 (1959).

${ }^{3}$ H. Knoepfel and D. A. Spong, Nucl. Fusion 19, 785 (1959).

${ }^{4}$ J. W. Connor and R. J. Hastie, Nucl. Fusion 15, 415 (1975).

${ }^{5}$ Yu. A. Sokolov, JETP Lett. 29, 218 (1979).

${ }^{6}$ P. Helander, L.-G. Eriksson, and F. Andersson, Plasma Phys. Controlled Fusion 44, B247 (2002).

${ }^{7}$ R. D. Gill, Nucl. Fusion 33, 1613 (1993).

${ }^{8}$ R. D. Gill, B. Alper, A. W. Edwards, L. C. Ingesson, M. F. Johnson, and D. J. Ward, Nucl. Fusion 40, 163 (2000).

${ }^{9}$ R. D. Gill, B. Alper, M. de Baar, T. C. Hender, M. F. Johnson, and V. Riccardo, Nucl. Fusion 42, 1039 (2002).

${ }^{10}$ R. Yoshino and S. Tokuda, Nucl. Fusion 40, 1293 (2000).

${ }^{11}$ R. M. O. Galvao, Yu. K. Kuznetsov, I. C. Nascimento, E. Sanda, D. O. Campos, A. G. Elfimov, J. I. Elizondo, A. N. Fagundes, A. A. Ferreira, A. M. M. Fonseca, E. A. Lerche, R. Lopez, L. F. Ruchko, W. P. de Sa, E. A. Saettone, J. H. F. Severo, R. P. da Silva, V. S. Tsypin, R. Valencia, and A. Vannucci, Plasma Phys. Controlled Fusion 43, 1181 (2001).

${ }^{12}$ Yu. K. Kuznetsov, R. M. O. Galvao, V. Bellintani, Jr., A. A. Ferreira, A. M. M. Fonseca, I. C. Nascimento, L. F. Ruchko, E. A. O. Saettone, V. S. Tsypin, and O. C. Usuriaga, Nucl. Fusion 44, 631 (2004).

${ }^{13}$ B. Esposito, L. Bertalot, Yu. A. Kaschuch, D. V. Portnov, and J. R. Martin-Solis, Nucl. Instrum. Methods Phys. Res. A 476, 522 (2002).

${ }^{14}$ Z. Y. Chen, B. N. Wan, S. Y. Lin, Y. J. Shi, L. Q. Hu, and T. P. Ma, Nucl. Instrum. Methods Phys. Res. A 560, 558 (2006).

${ }^{15}$ E. Farshi, R. Amrollahy, A. V. Bortnikov, N. N. Brevnov, Yu. V. Gott, and V. A. Shurygin, Plasma Phys. Rep. 27, 545 (2001).

${ }^{16}$ L. Rodriguez-Rodrigo and F. Castejon, Phys. Rev. Lett. 74, 3987 (1995).

${ }^{17}$ L. Rodriguez-Rodrigo, A. Rodriguez-Yunta, F. Castejon, J. Vega, C. Pardo, and A. P. Navarro, Nucl. Fusion 34, 649 (1994). 